\title{
Challenges to Inter-Satellite Communication System: A Review
}

\author{
Abdallah Ahmad Shatnawi \\ ENAC, School of \\ Computer and Communication \\ Engineering (SCCE) Universiti \\ Malaysia Perlis (UniMAP)
}

\author{
Mohd Nazri Mohd Warip \\ ENAC, School of \\ Computer and Communication \\ Engineering (SCCE) Universiti \\ Malaysia Perlis (UniMAP)
}

\begin{abstract}
Inter-Satellite communication link is the valuable technique to establish the link between satellites. It is revolutionary technique in which laser is used to transmit data from one side to another. This paper briefly explains overview of this technology. Furthermore, various challenges to this technology are also reported.
\end{abstract}

\section{General Terms}

Optical wireless communication, Satellite Communication

\section{Keywords}

InterSatellite Communication Link (Is-OWC), Transmitting Pointing Errors, Receiving Pointing Error

\section{INTRODUCTION}

Optical remote communication is considered appropriate for advanced indoor and outdoor broadband remote applications ranging from short-run remote communication systems to last-mile connection systems with access between end clients, existing fiber optic communications, and inter-space laser connections [1]. Indoor optical remote communication is known as remote infrared communication whereas outdoor optical remote communication is known as free space optical (FSO) communication. One of the most significant applications of FSO is that of inter-satellite optical wireless communication (Is-OWC) system transmitted between satellite and space interchanges within a short span. In case of the applications of wireless infrared communication, nondirected links without any transmitter-receiver alignment are required. These links are categorized as line-of-sight (LOS) and diffuse links. LOS links require a specified path for hassle-free communication whereas diffuse links require multiple optical paths from surface reflections. Is-OWC requires directed LOS and point-to-point laser links between transmitter and receiver via the atmosphere. OWC technology provides potentials of broadband communication capacity with unlicensed optical wavelengths. But disparities in temperature and atmospheric pressure result in refractive index variations over the transmission path, which further leads to spatial and temporal variations in the optical intensity on the receiver resulting in fading [2]. Further, the faded links can adversely affect system performance resulting in increased bit error rate (BER) and transmission delays.

The application of Is-OWC can be used for inter-satellite communication in similar or distinctive circles with the lightwave transmission of $3 \times 108 \mathrm{~m} / \mathrm{s}$ through which information can be transmitted with larger data and less constriction [3]. Higher quantity of information can be sent to a longer distance by using optical connection over radio frequency (RF) technology with fewer payloads. Moreover, OWC systems use RF wavelength which is more suitable than lasers in terms of beam-width, resulting in lower attenuation [4]. Is-OWC systems are quickly deployable and provide secure communication frameworks which are compatible with any advanced sensor communication system.

In this paper, a review is done on previous works in era of IsOWC systems. Furthermore challenges to Is-OWC are also reported. The rest of paper is divided as: Section II describes background work; Section III describes the various challenges in Inter-Satellite communication system followed by Section IV which concludes conclusion.

\section{BACKGROUND STUDY (RELATED WORK)}

The concept of laser technology to space communications was developed in the early 1960s in order to secure communication between a satellite and a submarine. In the present course of time, the application has witnessed remarkable technical advancement particularly in optical wireless communication as implemented widely by government authorities, corporates, and higher academics. The use of precision pointing and tracking control subsystems in optical inter-satellite communication was proposed in 1990 [5]. Moreover, the study identified disturbance effects and developed appropriate models to resolve them. While performing simulations for the control system, it was compared with a rate-integrating based system and the results rejected superior disturbance of a complimentary filter-based precision pointing and tracking subsystem. However, these two systems were unable to meet the 0.5 - $\mu$ murad pointing restriction enforced by optical inter-satellite communication links.

In order to minimize FWM-induced crosstalk, a technique was developed for channel frequency allocation [6]. The study found that when the system bandwidth is expanded, unequal channel separations are available without any imposition of four-wave mixing product on the channels. The study also performed simulation in a system of 10 -channel with $10 \mathrm{Gbps}$ per channel in order to probe the effectiveness of the technique.

Another study discussed the probability of high speed optical satellite network construction as a part of integrated spaceterrestrial network [7]. The study described the utility of optical wireless inter-satellite links for global networking with the creation of local area networks and wide area networks across the globe. Inter-satellite links can also be used for connecting remote sensing satellites, submarines and ships, airplanes, and space-crafts. The current study aims to design an advanced block diagram of inter-satellite optical transceiver which includes tracking and precision pointing system between the transceiver and other satellites. 
Quantum cryptography can also be employed in inter-satellite links [8]. The study used the following equation to measure the attenuation (A) for inter-satellite and satellite-to-earth links:

$A=\frac{L^{2}\left(\theta_{T}^{2}+\theta_{a t m}^{2}\right)}{D_{R}^{2}} \frac{1}{T_{T}\left(1-L_{P}\right) T_{R}} 10^{\frac{A_{a t m}}{10}}$

As atmospheric turbulences do not affect the inter-satellite communication link, it can further lead to attenuation reduction as shown in the equation below:

$A=\frac{L^{2} \theta_{T}^{2}}{D_{R}^{2}} \frac{1}{T_{T}\left(1-L_{P}\right) T_{R}}$

The study measured attenuation for LEO-LEO and GEO-GEO inter-satellite links and found that longer transmission distance requires larger optical antenna.

Another study used laser as a beacon and a transmitter in a pointing control system for inter-satellite laser communication and ranging link [9]. The traffic capacity of the link can be maximized by controlling the beamwidth from broad (in the acquisition stage) to narrow (in the tacking stage). The requirements of laser link pointing control are difficult to achieve by the beam pointing precision. Inter-satellite laser link in orbits can be established and maintained by fast and precise pointing control. The study proposed a hierarchical two-level system control in which the lower level controls the optic head gimbals of the electro-optical transceiver and the higher level controls the beam-width and direction with a closed loop. The study provides a detailed description of control algorithms and representative simulations used for pointing acquisition control in inter-satellite information transmission. Optical inter-satellite network can also be developed using wavelength division multiplexing (WDM) technology consisting of a fixed node, high altitude platform (HAP) of $30 \mathrm{Km}$ from the earth's surface, and a mobile node on a LEO orbit communicating between HAP and eight LEO micro satellites [10]. Micro satellites can efficiently cover target area while transferring data continuously from slave nodes to the master node and thus increasing communication capacity. HAP is used for configuring ad-hoc network to monitor emergency application including natural or manmade disasters. However, the signal can suffer from the Doppler Effect due to inter-satellite motion which affects the system performance. The components of the designed optical subsystem are: semiconductor laser, ITU grid transmission, EDFA preamplifier, and the direct detection receiver scheme. The study evaluated signal-to-noise ratio for the received optical power along with EDFA achievements and intersatellite distance for two modulation formats: OOK and DPSK.

Another study examined error performance of heterodyne differential phase-shift keying (DPSK) OWC system under intensity-fluctuated conditions such as turbulence-induced fading and path loss [11]. The study investigated random attenuation of the propagation channel and the bit-error rate (BER) performance regarding the fading effects of atmospheric turbulence. It also studied error performance of nonlinear optimization in order to find the optimum beamwidth for the minimum BER of a signal-to-noise ratio value. Laser satellite communication can be used for inter-satellite and satellite-to-ground communications [12]. With the growing number of satellites orbiting the earth every year, inter-satellite network can provide a communication method for them. Moreover, laser communication can connect satellites with a data rate up to several Gbps without any lengthy fiber. The study focused on the OWC link performance for data transmission between Low Earth Orbit (LEO) satellites while examining bit rates, wavelength, input power, and inter-satellite link distance.

While examining the outage behavior of optical inter-satellite communication links, a study found that long wavelength provides better performance than shorter wavelength [13] The study analyzed the following parameters: source-induced and statistically correlated fading, transmitter variations, and selection and equal gain combining. It found that when correlation coefficient is less than 0.5 , combining performs better; more particularly, EGC scheme performs better than SC scheme.

Another study proposed the design of an ultra-high bit-rate (400 Gbps) inter-satellite optical wireless communication (IsOWC) system which was a non-diffused link used for quadrature phase-shift keying (QPSK) modulation [14]. The study examined the system performance, with various parameters such as Q-factor, eye opening, and bit-error rate, while observing coverage distance of $30 \mathrm{dBm}$ input power at the bit rates of $400 \mathrm{Gbps} / 4767 \mathrm{Km}, 160 \mathrm{Gbps} / 7542 \mathrm{Km}$, and $100 \mathrm{Gbps} / 9532 \mathrm{Km}$. The study presented the maximum bitrate for inter-satellite links at low-earth, medium-earth, and geostationary-earth orbits.

Another study investigated the BER performance for DQPSKmodulated OWC system over Gamma-Gama turbulence channels [15]. The study derived a closed-form BER expression and found that the smaller channel parameter can determine the diversity order of the system.

Another study found that inter-satellite link (ISL) can be implemented between satellites distanced by $1000 \mathrm{Km}$ at a data rate of $2.5 \mathrm{Gbps}$ with or without square root module (SM) [16]. SM module can be used for achieving BER with improved SNR ratio while transmitting less power for 2.5 Gbps data over ISL link of $1000 \mathrm{Km}$ at $1550 \mathrm{~nm}$ wavelength [17].

Gamma-Gamma turbulence channels can be combined with pointing errors in subcarrier intensity-modulated OWC system for examining BER performance [18]. The study used M-ary phase-shift keying, non-coherent frequency-shift keying, and differential phase-shift keying for observing error performance and found that pointing errors can adversely affect the error rate and outage probability performance of the system.

Another study designed an inter-satellite OWC system for establishing ISL of $1000 \mathrm{Km}$ length between satellites at 2.5 Gbps data rate [19]. Two different operating wavelengths were measured for the system performance: $1550 \mathrm{~nm}$ and 850 $\mathrm{nm}$. The study found that the ISL with acceptable BER can be achieved at the operating wavelength of $850 \mathrm{~nm}$ which uses less power transmission than the wavelength of $1550 \mathrm{~nm}$.

Another study used polarization interleaving (PI) technique to reduce FWM crosstalk [20]. While analyzing FWM behavior and WDM performance, the study conducted simulation with $100 \mathrm{GHz}$ channel spacing at various power values and 60 Gbps data rate. The study found that the use of PI technique attenuated FWM power to $-64 \mathrm{dBm}$ and gained a BER of $4.86 \times 10-21$ for the WDM system at the sixth channel (192 $\mathrm{THz}$ ), thus making the proposed system superior for moderating FWM crosstalk.

Pulse position modulation (PPM) scheme was used by another study to analyze the performance of a $2.5 \mathrm{Gbps}$ Is-OWC 
system [21]. The study used a number of parameters such as transmission range and power, receiver aperture diameter, and RIN value, and found that electro-optical SNR with transmission range decreases for higher transmission wavelength. The increase in the receiver aperture further increases the electro-optical SNR whereas increase in transmission range further decreases electro-optical SNR. Moreover, increase in transmission power further increases electro-optical SNR. The use of PPM scheme led the system achieve a distance of $2500 \mathrm{Km}$ at acceptable BER in HAPsatellite links. Furthermore, Wavelength division multiplexing in conjunction with polarization interleaving is adopted for inter-satellite communication system which shows prior improvement as compared to previous works [22-24].

\section{CHALLENGES TO IS-OWC LINK}

Figure 1 shows the broad categorization of optical communication systems. There are two major categories: fiber communication and optical wireless communication. Fiber communication uses fiber as medium or channel for communication whereas optical wireless communication transmits information without using fiber. Optical wireless communication is further divided into two categories: IsOWC and free space optics (FSO). Is-OWC is used for communication between satellites and FSO for ground or terrestrial communication.

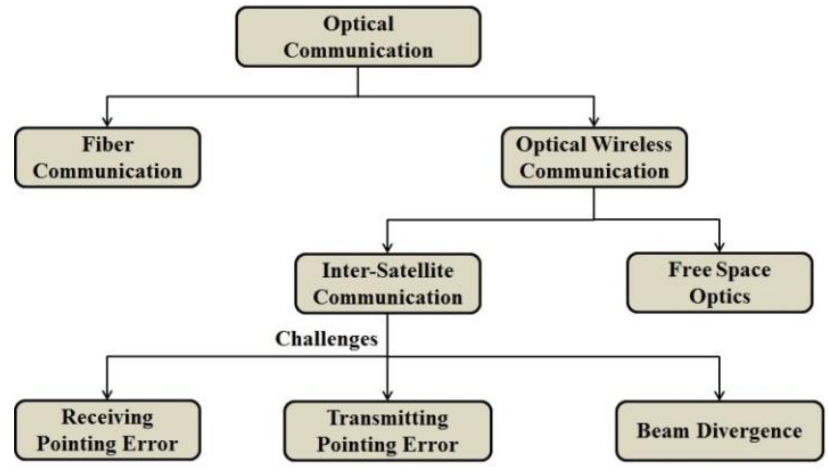

Fig.1 Classification of Optical Communication

Previous studies on Is-OWC have reported certain challenges such as beam divergence, and receiving and transmitting pointing errors which further limit transmission distance and capacity [25]. When the transmitter and the receiver are not aligned, it leads to power reduction at the receiver side. These further results in pointing losses as shown by the equation below [26]:

$L_{\text {pointing }}=4.3229\left(\frac{\phi_{e}}{\Omega_{0}}\right)^{2}$

In the above equation, pe refers to the boundary angle of diffraction which is the limited beam of the transmitter. Beam divergence refers to spreading of beam during its propagation from transmitter to receiver. These challenges must be considered by researchers during the design of inter-satellite communication system.

\section{CONCLUSION}

Is-OWC is the revolutionary technique which can establish the communication link between satellites through lasers. No doubt, Is-OWC communication has lot of rigorous advantages, but there are some challenges also which causes poor performance of its link. Turbulences such as transmitting pointing errors, receiving pointing errors, beam divergences etc. results in increase of attenuation which causes shut down of inter-satellite communication link. These turbulences must be taken under consideration while designing Is-OWC transmission system.

\section{REFERENCES}

[1] R. KANMANI and K. SANKARANARAYANAN, "Study of Wireless Optical CDMA LAN in Indoor Environment," system, vol. 2, 2013.

[2] B. Deng, S. Zhao, Y. Li, X. Zhang, and Z. Cheng, "The design of inter-satellite laser link interface model based on standardized transfer mode," in Optical Communications and Networks (ICOCN), 2015 14th International Conference on, 2015, pp. 1-3.

[3] N. Kumar, "Enhanced performance analysis of intersatellite optical-wireless communication (IsOWC) system," Optik-International Journal for Light and Electron Optics, vol. 125, pp. 1945-1949, 2014.

[4] R. Q. Shaddad, A. Mohammad, S. A. Al-Gailani, A. AlHetar, and M. A. Elmagzoub, "A survey on access technologies for broadband optical and wireless networks," Journal of Network and Computer Applications, vol. 41, pp. 459-472, 2014.

[5] V. Trent, M. Greene, and S. Hung, "Precision pointing error analysis in a satellite optical communication optical system," in System Theory, 1990., Twenty-Second Southeastern Symposium on, 1990, pp. 190-194.

[6] F. Forghieri, R. Tkach, and A. Chraplyvy, "WDM systems with unequally spaced channels," Lightwave Technology, Journal of, vol. 13, pp. 889-897, 1995.

[7] V. W. Chan, "Optical satellite networks," Journal of Lightwave Technology, vol. 21, p. 2811, 2003.

[8] M. Pfennigbauer, W. Leeb, M. Aspelmeyer, T. Jennewein, and A. Zeilinger, Free-space optical quantum key distribution using intersatellite links: na, 2003.

[9] M. Guelman, A. Kogan, A. Livne, M. Orenstein, and H. Michalik, "Acquisition and pointing control for intersatellite laser communications," Aerospace and Electronic Systems, IEEE Transactions on, vol. 40, pp. 1239-1248, 2004

[10] S. Betti, V. Carrozzo, and G. Parca, "Optical Intersatellite hybrid network links based on WDM technology," in Transparent Optical Networks, 2008. ICTON 2008. 10th Anniversary International Conference on, 2008, pp. 209-212.

[11] H. G. Sandalidis, T. A. Tsiftsis, and G. K. Karagiannidis, "Optical wireless communications with heterodyne detection over turbulence channels with pointing errors," Journal of lightwave technology, vol. 27, pp. 4440-4445, 2009

[12] A. H. Hashim, F. D. Mahad, S. M. Idrus, and A. S. M. Supa'at, "Modeling and performance study of intersatellite optical wireless communication system," in Photonics (ICP), 2010 International Conference on, 2010, pp. 1-4.

[13] X. Liu, "Outage Behavior of a MISO Wireless Optical Link with Pointing and Tracking Errors," in Communications (ICC), 2011 IEEE International Conference on, 2011, pp. 1-5. 
[14] B. Patnaik and P. K. Sahu, "Inter-satellite optical wireless communication system design and simulation," Communications, IET, vol. 6, pp. 2561-2567, 2012.

[15] X. Song, Z. Hassan, and J. Cheng, "Subcarrier DQPSK modulated optical wireless communications in atmospheric turbulence," Electronics letters, vol. 48, p. 1224,2012

[16] V. Sharma and N. Kumar, "Improved analysis of 2.5 Gbps-inter-satellite link (ISL) in inter-satellite opticalwireless communication (IsOWC) system," Optics Communications, vol. 286, pp. 99-102, 2013.

[17] V. Sharma and N. Kumar, "Modeling of 2.5 Gbpsintersatellite link (ISL) in inter-satellite optical wireless communication (IsOWC) system," Optik-International Journal for Light and Electron Optics, vol. 124, pp. 6182-6185, 2013.

[18] X. Song, F. Yang, and J. Cheng, "Subcarrier intensity modulated optical wireless communications in atmospheric turbulence with pointing errors," Journal of Optical Communications and Networking, vol. 5, pp. 349-358, 2013.

[19] N. Kumar and D. Rana, "Enhanced performance analysis of inter-aircraft optical-wireless communication (IaOWC) system," Optik-International Journal for Light and Electron Optics, vol. 125, pp. 486-488, 2014.

[20] H. J. Abd, N. Din, M. Al-Mansoori, F. Abdullah, and H. Fadhil, "Mitigation of FWM crosstalk in WDM system using polarization interleaving technique," in Photonics
(ICP), 2013 IEEE 4th International Conference on, 2013, pp. 117-119.

[21] N. Kumar, "2.50 Gbit/s optical wireless communication system using PPM modulation schemes in HAP-tosatellite links," Optik-International Journal for Light and Electron Optics, vol. 125, pp. 3401-3404, 2014.

[22] Chaudhary, Sushank, and Abhishek Sharma. "6 x 20Gbps Long Reach WDM-PI based High Altitude Platform Inter-Satellite Communication System." International Journal of Computer Applications 122.22 (2015).

[23] Chaudhary, Sushank, Abhishek Sharma, and Neha Chaudhary. "6× 20 Gbps Hybrid WDM-PI Inter-satellite System under the Influence of Transmitting Pointing Errors." Journal of Optical Communications (2016).

[24] Chaudhary, Sushank, and Saurabh Sharma. "Role of Turbulences in WDM-Polarization Interleaving Scheme based Inter-Satellite Communication System." International Journal of Computer Applications 104.10 (2014).

[25] Chaudhary, Sushank, and Angela Amphawan. "The role and challenges of free-space optical systems." Journal of Optical Communications 35.4 (2014): 327-334.

[26] H. G. Sandalidis, T. A. Tsiftsis, and G. K. Karagiannidis, "Optical wireless communications with heterodyne detection over turbulence channels with pointing errors," Journal of lightwave technology, vol. 27, pp. 4440-4445, 2009. 